Schroeder.-Tuo Cases of Severe Acute Purulent Median Otitis, caused by "Schneeberger" Smuff-powder. München: "Med. Woch.," November 25, 1902.

The first case was a man, aged thirty, who snuffed a pinch of Thuringean "Schneeberger" to relieve a severe cold in the head. He did not sneeze, but within ten minutes felt severe pain in the left ear, and developed the signs of hæmorrhagic median otitis. Posterior rhinoscopy revealed intumescence of the left half of the naso-pharyngeal cavity and the Eustachian cushion. Perforation followed, and healing took place in four weeks. In this case there was a spur on the left side of the septum, which closed the olfactory region, so that the powder went straight through the inferior meatus to the Eustachian tube.

In the second case, a boy, aged fourteen, suffering from ozœna, was recommended by his father to snuff up this same powder with the object of clearing " not only the nose, but also the brain." The result was " kolossal," but not exactly what was desired. Pain in both ears came on in a few hours, and the boy went through a severe attack of purulent otitis.

It appears that this powder consists chiefly of the powdered root of the iris, but the specimen employed probably contained veratrum.

Kessel and Haug have reported cases of acute ear disease resulting from ordinary snuff.

Dundas Grant.

\title{
LARYNX, Etc.
}

Delavan, Bryson. - The Results of Treatment of Laryngeal Cancer b!l Means of the X Rays. " "Laryngoscope," December, 1902.

So far as the author has been able to ascertain, not a single case of carcinoma of the larynx has been reported as cured by X-ray treatment. In an average case, however, where the progress of the disease has not been rapid, and where a few days must necessarily elapse between the defnite diagnosis of the disease and the performance of any operative measure for its relief, the author considers that it would be quite justifiable to submit the patient to treatment by means of the $\mathrm{X}$ rays.

W. Milligan.

\section{EAR.}

Dowling, J. Ivimey.-The Ear Complications of la Grippe. "The Journal of Ophthalmology, Otology, and Rhinology," May, 1902.

The author considers the chief predisposing factor in the production of these troubles to be an unhealthy condition of the nares and nasopharynx; with these in a normal condition influenza would be less likely to induce auditory mischief. He divides the complications into immediate and remote. The immediate complications vary from an acute hyperæmia of the tympanum and its adnexa to a severe otitis media and mastoiditis. The remote, which set in from a few months to a year or two, consist for the most part of a hyperplastic inflammation of the Eustachian tubes with resulting stenosis, and are evidenced by labyrinthine symptoms-as tinnitus and vertigo-thickened and retracted membranes, and rotated mallei. 